\title{
Desynchronization of jammed oscillators by avalanches
}

\author{
William Gilpin $\odot^{*}$ \\ Quantitative Biology Initiative, Harvard University, Cambridge, Massachusetts 02138, USA; \\ Department of Physics, The University of Texas at Austin, Austin, Texas 78712, USA; \\ and Oden Institute for Computational Engineering and Sciences, The University of Texas at Austin, Austin, Texas 78712, USA
}

(Received 2 December 2020; accepted 17 May 2021; published 11 June 2021)

\begin{abstract}
Synchrony is inevitable in many oscillating systems-from the canonical alignment of two ticking grandfather clocks, to the mutual entrainment of beating flagella or spiking neurons. Yet both biological and man-made systems provide striking examples of spontaneous desynchronization, such as failure cascades in alternating current power grids or neuronal avalanches in the mammalian brain. Here, we generalize classical models of synchronization among heterogenous oscillators to include short-range phase repulsion among individuals, a property that allows the emergence of a stable desynchronized state. Surprisingly, we find that our model exhibits self-organized avalanches at intermediate values of the repulsion strength, and that these avalanches have similar statistical properties to cascades seen in real-world systems such as neuronal avalanches. We find that these avalanches arise due to a critical mechanism based on competition between mean-field recruitment and local displacement, a property that we replicate in a classical cellular automaton model of traffic jams. We exactly solve our system in the many-oscillator limit, and we obtain analytical results relating the onset of avalanches or partial synchrony to the relative heterogeneity of the oscillators and their degree of mutual repulsion. Our results provide a minimal analytically tractable example of complex dynamics in a driven critical system.
\end{abstract}

DOI: 10.1103/PhysRevResearch.3.023206

\section{INTRODUCTION}

Spontaneous synchronization occurs in diverse systems such as robotic swarms, power grids, neuronal ensembles, and even social networks [1-5]. Globally coupled phase oscillators represent a basic, tractable paradigm for understanding synchronization, formulating the phenomenon as the gradual mutual entrainment of individual oscillators due to the compounded effect of continuous weak interactions [4]. This paradigm stems from pioneering midcentury work by Winfree [6] and Kuramoto [7], who established the universality of minimal phase oscillator models for understanding synchronization in a wide variety of biological processes. Subsequent work has established general results for the onset of synchronization in phase oscillators, and results from these simple, tractable models have been successfully applied to the study of biological systems spanning from brain waves, to bacterial signaling, to swimming microorganisms [8-12]. However, while the theory of phase oscillators is well established, in recent years a variety of novel physical phenomena have been discovered in variants of phase oscillator models [13-16], including chimera states $[17,18]$, glasslike relaxation mediated by a "volcano" transition [19], and oscillation death via broken rotational symmetry [20]. These results underscore

\footnotetext{
*wgilpin@fas.harvard.edu

Published by the American Physical Society under the terms of the Creative Commons Attribution 4.0 International license. Further distribution of this work must maintain attribution to the author(s) and the published article's title, journal citation, and DOI.
}

that even simple abstractions of real-world oscillators can display surprisingly complex dynamics.

Many such discoveries are framed in terms of their effects on the synchronous state, which represents a globally stable solution of the underlying dynamical equations. However, many real-world oscillator populations, such as neuronal ensembles, exhibit a maximally desynchronous state, in which the phases of individual oscillators become evenly spaced apart on the unit circle $[21,22]$. Such dynamics are achievable, in principle, by introducing negative couplings into standard phase oscillator models [23,24]; alternative approaches include introducing phase offsets or time delays in the interactions among oscillators, or introducing specific pairwise couplings among oscillators that embed them on a complex graph $[3,25,26]$. The tunability of synchronization in these models introduces the broader question of whether unique phenomena can occur at the critical intermediate point between the synchronous and maximally desynchronous states.

Here, we investigate this regime by introducing a minimal, tractable generalization of classical oscillator models, which allows a smooth transition between full synchrony and maximal desynchrony. Our model is based on short-range steric interactions observed in swarms and active matter, and it bears resemblance to local inhibitory effects observed in real-world ensembles of interacting neurons. Our model exhibits a surprising phenomenon consisting of self-organized avalanches, in which the system seemingly approaches synchrony, only to abruptly desynchronize at quasirandom intervals. Despite appearing in a minimal phase model, these avalanches have similar statistical properties and scaling exponents to avalanches observed in real-world oscillator networks, including neuronal ensembles, financial markets, and power grids 
[27-29]. We trace these avalanches to a critical mechanism driven by competition between large-scale attraction of oscillators to the mean field and small-scale rearrangements, which we recreate using a cellular automaton model inspired by self-organized criticality in traffic. Finally, we solve our model exactly in the many-oscillator limit and show that avalanches in the system originate in the amplification of noiselike excitations provided by local rearrangements.

\section{APPROACH: THE KURAMOTO MODEL WITH SHORT-RANGE REPULSION}

Our model consists of nonidentical phase oscillators with global attraction through sinusoidal coupling; we modify this model by adding an additional term corresponding to shortrange repulsion among oscillators with proximal phases,

$$
\dot{\theta}_{j}=\omega_{j}+\frac{1}{N} \sum_{k=1}^{N}\left(K \sin \left(\theta_{k}-\theta_{j}\right)-\frac{d}{d \theta_{j}} V\left(d\left(\theta_{j}, \theta_{k}\right)\right)\right),
$$

where the interaction $V(\cdot)$ represents a short-range repulsion with amplitude $V_{0} \equiv V(0)$ and width $2 \pi / N$; the width scales inversely with $N$ in order to ensure short-ranged interactions. The angular distance function $d\left(\theta_{j}, \theta_{k}\right)$ corresponds to the signed shortest distance between two angles along the unit circle. The individual oscillator frequencies $\omega_{j}$ are sampled from a probability distribution $g\left(\omega_{j}\right)$; following previous work, we use a Cauchy distribution with mean frequency $\bar{\omega}$ and width $\sigma_{\omega}$. Below we obtain comparable results for several choices of $V(\cdot)$.

When $V_{0}=0$, Eq. (1) becomes the standard Kuramoto model with global coupling and no phase offsets; this model exhibits solvable dynamics and synchronization when $K$ is large relative to $\sigma_{\omega}[4,30]$. The onset of synchronization can be readily observed via the dynamics of the Kuramoto order parameter,

$$
R(t) e^{i \Phi(t)} \equiv \sum_{k=1}^{N} e^{i \theta_{k}(t)},
$$

which reaches a stable steady-state $R=1$ when $K>0, \sigma_{\omega}=$ 0 . However, in the case of nonzero repulsion $V_{0}>0$, Eq. (1) admits another stable solution when $K=0$ and $\sigma_{\omega}$ is small, corresponding to equal spacing of oscillators along the unit circle $(R=0)$ due to steric interaction. Thus the relative values of $K$ and $V_{0}$ parametrize competition between global attraction to the mean field and local avoidance of clustering.

The repulsive interaction can be seen as an abstraction of steric interactions among individuals [31], as occurs in swarms of self-propelled oscillators [5,32]. Similar repulsive interactions occur in levitating colloidal particles, which produce collective dynamics reminiscent of those reported here [33]. Alternatively, in neurons, the repulsion term would correspond to a negative region in each neuron's phase-response curve; such a response could arise due to synaptic depression via local depletion of neurotransmitter reserves [34,35].

We contrast our approach with previous studies that generalize the Kuramoto model by introducing spatial effects through a nontrivial coupling matrix $K \rightarrow K_{i j}$. These studies include mixtures of purely excitatory and inhibitory interactions $[19,24,36]$, as well as complex spatial interaction kernels [37] or multiplexed interactions [38,39]—which can give rise to complex dynamics such as global desynchronization, local "ripple" states, and even chimera states. In contrast, our approach introduces short-range repulsion in each oscillator's phase-response curve, making it a particular realization of Kuramoto-Daido models, which extend the Kuramoto model to include generalized phase coupling interactions [40]. Recent works have explored the inclusion of higher-order harmonics, producing chaotic fluctuations in the order parameter $[13,41,42]$ and quasiperiodicity [43]. Here, we show that our choice of short-range repulsion produces particlelike interactions in the phase domain, such as finitesize effects, criticality, and jamming.

\section{RESULTS}

\section{A. Avalanche dynamics appear at intermediate coupling strengths}

Unexpectedly, at intermediate values of $K$ and $V_{0}$, the order parameter undergoes irregular oscillations, displaying epochs of rapid synchronization punctuated by gradual desynchronization events (Fig. 1). These fluctuations occur independently of the choice of repulsive kernel $V(\cdot)$; we show results for Gaussian, Cauchy, and triangular potentials in Fig. 2, although we otherwise focus on Gaussian repulsion for simplicity. Moreover, fluctuations persist over a range of repulsion radii, potential strengths, and ensemble sizes (see Appendix A). Qualitatively, oscillations of the order parameter (and frequency fluctuations of individual oscillators) resemble those of relaxation oscillators; intuitively, the repulsive term in Eq. (1) introduces a second timescale into the collective dynamics (the first being the synchronization rate). In relaxation oscillators, timescale separation between fast and slow manifolds produces complex dynamics; a similar effect occurs in integrate-and-fire neuron models. In our model, the second timescale arises directly from interaction with other oscillators.

These irregular oscillations are particularly striking given the global coupling among units. Self-organized quasiperiodicity has previously been reported in the phases of individual phase oscillators with nonlinear coupling [44]; in this system, when the coupling $K$ is too low to produce full synchrony, the mean field fails to entrain individual oscillators-however, $R(t)$ remains periodic. The behavior of Eq. (1) also differs from partial synchronization observed in chimera states, in which distinct synchronous and desynchronous subpopulations coexist within an ensemble of oscillators $[3,13,17,18,45,46]$. While chimera states can produce irregular oscillations of $R(t)$, the form and character of chimera states often arise specifically from the presence of mixed short- and long-ranged pairwise couplings. In contrast, the number of short-range interactions experienced by an oscillator subject to Eq. (1) varies continuously as other oscillators enter and exit its effective repulsive radius.

\section{B. Characterizing the critical mechanism}

We hypothesize that our observed cascades arise from a critical mechanism, due to (1) the universal long-term statistics of our system, which are invariant to the choice of 


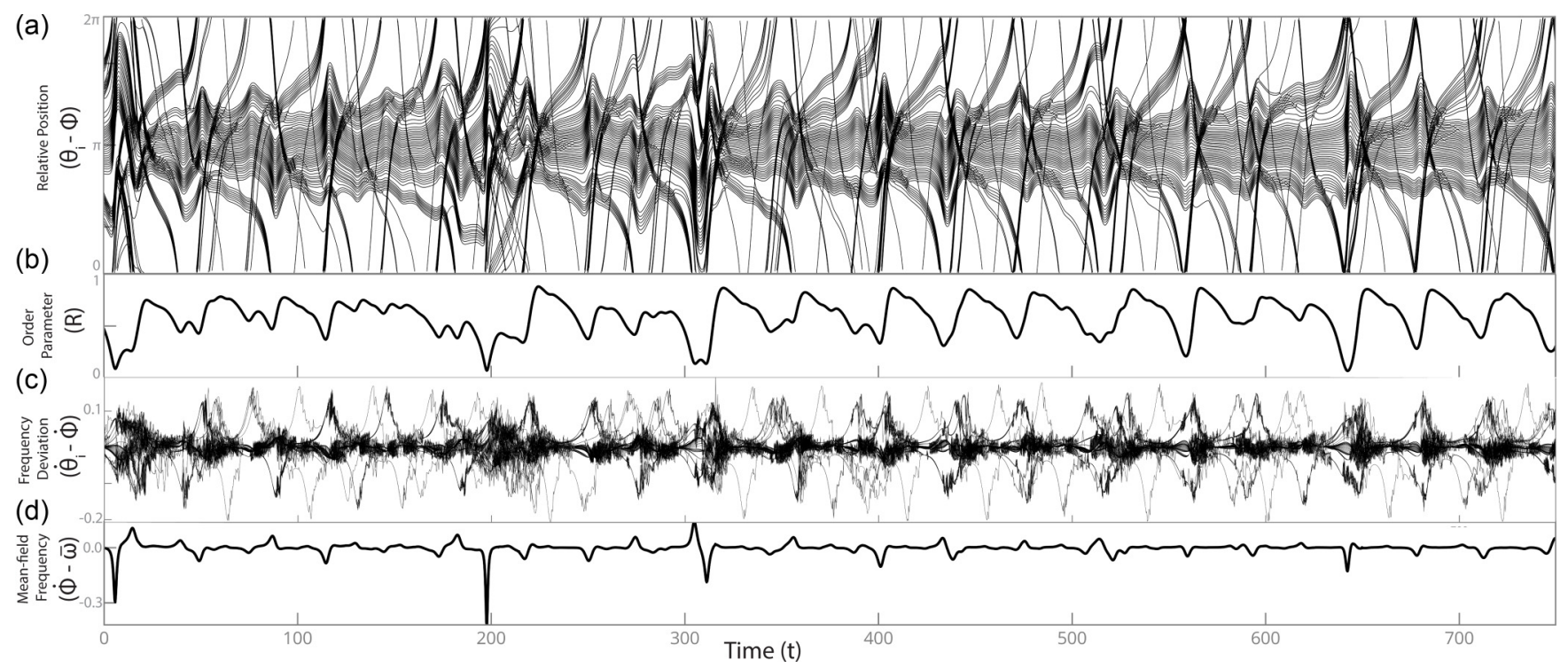

FIG. 1. Transient synchronization and desynchronization in a jammed oscillator ensemble. (a) A population of $N=60$ oscillators with short-range Gaussian repulsion, visualized in a frame rotating with the mean field. (b) The Kuramoto order parameter $R$; (c) the instantaneous values of individual frequencies; and (d) the mean frequency deviation from the population mean. For this figure and others (except where noted), $K=0.4, V_{0}=0.8, \sigma_{\omega}=0.2$.

interaction kernel; (2) the occurrence of cascades at intermediate regimes between fully dispersed and fully localized states; and (3) the lack of bistability in Eq. (1). We observe that each value of the order parameter $R(t)$ provides an upper bound for a range of possible values of the mean frequency $\Psi(t)$ [Fig. 2(d)], implying that cascades occur across a range of scales. Lending quantitative support to this hypothesis, we find that the power spectrum of the order parameter $R(t)$ exhibits $1 / f^{\alpha}$ decay, with $\alpha=1.5 \pm 0.08$ [Fig. 2(c)]; additionally, the higher-order order parameters

(a)

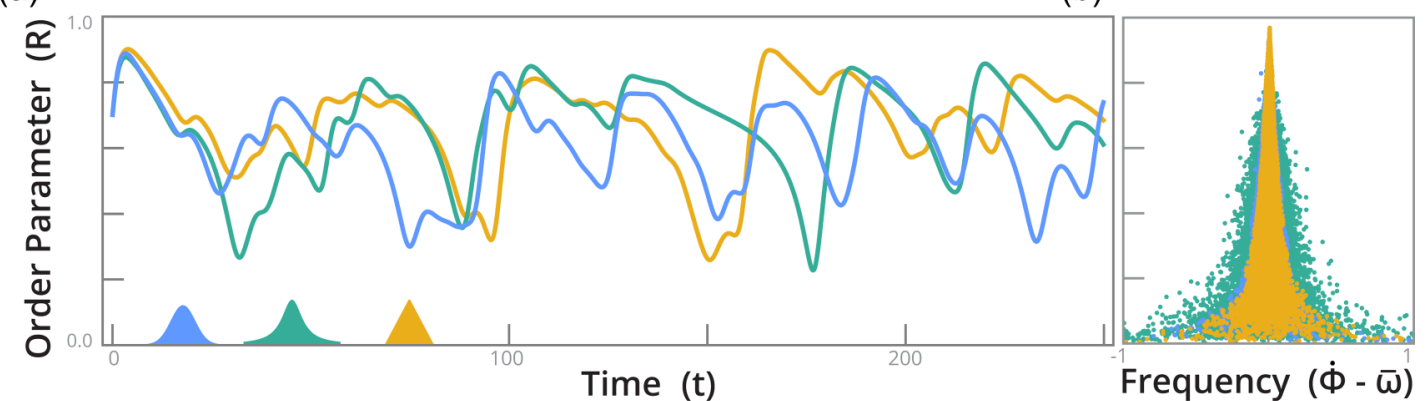

(c)

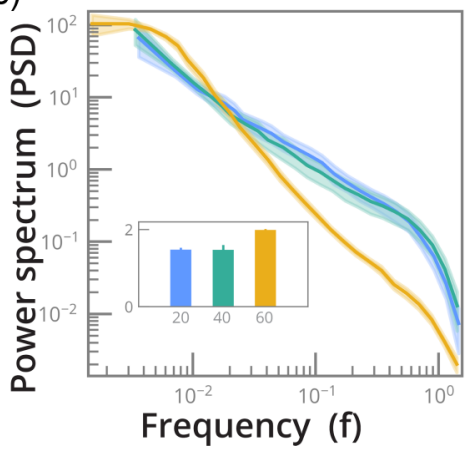

(d)

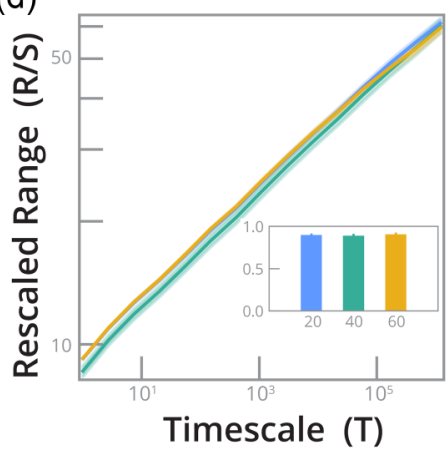

(e)

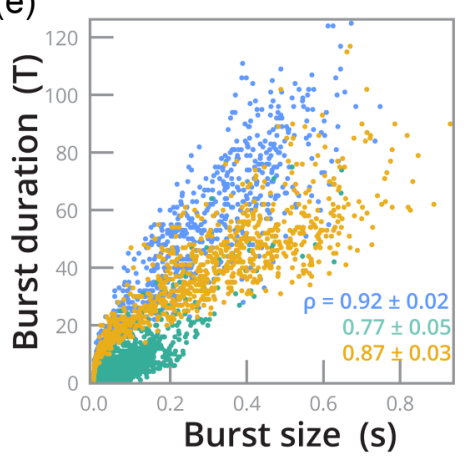

FIG. 2. Universal statistical properties of avalanches across different repulsive potentials. (a) Example dynamics of the order parameter $R$ for short-range repulsion given by Gaussian (blue), Cauchy (turquoise), and triangle (yellow) potentials (inset shapes). (b) $R$ vs the deviation between the mean field and the mean intrinsic frequency of the oscillators. (c) The power spectral density, with slopes corresponding to power-law decay exponents (inset). (d) A Hurst plot of the rescaled range vs measurement timescale, a measure of fractality for time series, with slopes corresponding to Hurst exponents (inset). (e) Avalanche duration vs size, annotated by Spearman correlation. All error ranges comprise bootstrapped standard deviations. 
$R_{n} \equiv \sum_{j=1}^{N} e^{i n \theta_{j}(t)}, n>1$, exhibit more gradual decay at low frequencies, indicating stronger temporal correlations in the clustering of oscillators over long timescales. Similar scaling exponents occur in several systems with self-organized criticality, including sandpile models, granular relaxation, and experimental measurements of "stick-slip" friction [47-50]. Further evidence of criticality comes from the scaled range versus time interval, which shows a straight-line trend indicative of self-similarity over five orders of magnitude [Fig. 2(d)]. The slope corresponds to a Hurst exponent $H=0.91 \pm$ $0.01 \approx 1$, indicating long-time correlations consistent with criticality [51]; moreover, experimental work has observed that $H \approx 1$ may indicate criticality even in finite systems [52]. Consistent with a critical mechanism, we find strong positive correlation $(0.92 \pm 0.02,954$ events) between the size of individual drops in $R$ (peak-to-trough amplitude) and the duration of each event (peak-to-peak distance) - a sign of a critical relaxation mechanism driving desynchronization [Fig. 2(e)] $[47,48,53]$.

We do not rule out whether avalanches are transient within our system, although we note that transience would not preclude criticality, because nonconservative systems can be transiently critical. For example, semidisordered chimera states originally observed in discrete oscillator ensembles were later found to be transient, but very long-lived $[3,54,55]$. However, we note that when $\sigma_{\omega}=0$, the avalanches are necessarily transient because Eq. (1) can be written as the gradient of a potential [56]; however, we observe in practice that fluctuations rarely cease when $N>20$ or $\sigma_{\omega}>0.01$, introducing the possibility that our phenomenon is either stable or a "supertransient" that scales sharply with system size [54]. In any case, the persistence of avalanches, and the wide range of parameter values over which they occur, suggest that our observed dynamics are an important phenomenon for systems of the form of Eq. (1) — especially real-world systems with noise or other driving that could continuously retrigger cascades.

Having observed long-term statistical properties consistent with self-organized criticality, we next consider the microscopic mechanism of criticality in our system. Close inspection of individual oscillators during a single synchronizationdesynchronization cycle reveals that rearrangements drive the onset of avalanches [Fig. 3(a)]. Intuitively, attraction of oscillators towards the mean field causes an exponential increase in local density in $\theta$ space. This clustering increases the steric pressure on synchronized oscillators, increasing the probability that two oscillators will overlap sufficiently to either exchange positions or exert a joint force on a third oscillatorthereby triggering a cascade of oscillator rearrangements and transient desynchronization. We quantify this effect by computing the net repulsive force acting on oscillator $j$, due to the cumulative effect of other oscillators falling within its radius of interaction,

$$
\mathcal{F}_{j}=-\sum_{k=1}^{N} \frac{d}{d \theta_{j}} V\left(\theta_{j}, \theta_{k}\right),
$$

which is proportional to the gradient of the local density of oscillators: An oscillator with an equal number of neigh-
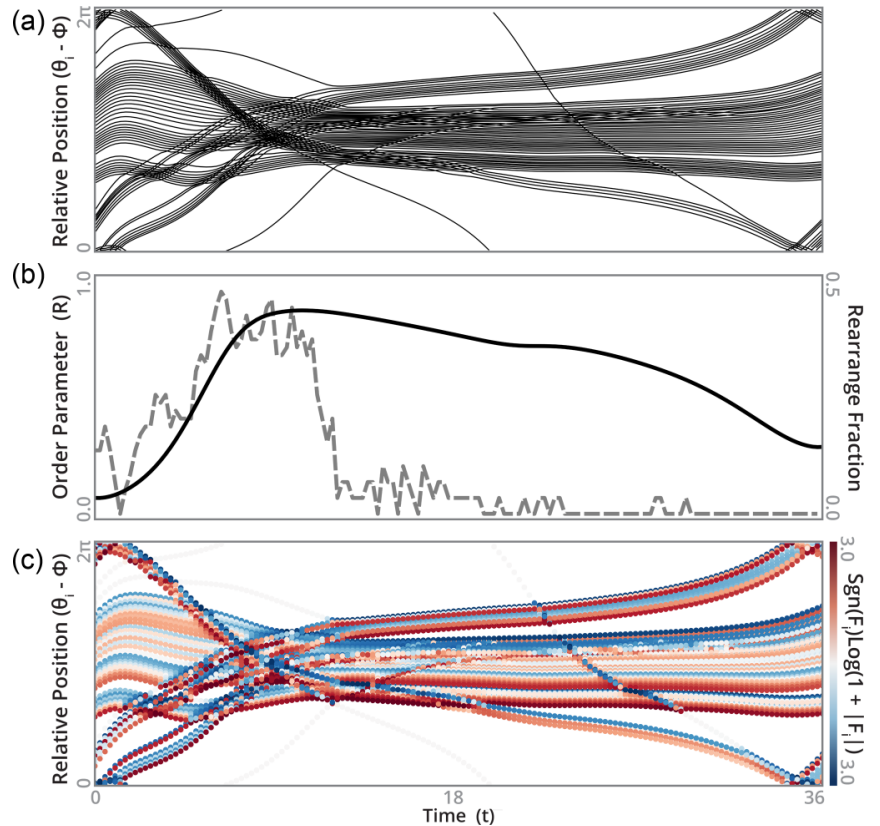

FIG. 3. Microscale oscillator rearrangements trigger avalanches. (a) Positions of oscillators during a typical synchronizationdesynchronization cycle. (b) The order parameter $R$ (left axis) and the fraction of rearrangements per time step (right axis). (c) The oscillator positions colored by net repulsive force $\mathcal{F}$.

bors falling on its right and left sides experiences zero net force. Figure 3(c) shows $\mathcal{F}_{j}$ overlaid as coloration of the traces in Fig. 3(a). As an oscillator $j$ exchanges positions, the sign of $\mathcal{F}_{j}$ fluctuates, giving rise to short-timescale dynamics necessary for criticality. Additionally, the magnitude of $\left|\mathcal{F}_{j}\right|$ increases during periods in which the order parameter is large, underscoring the role of local force buildup in producing avalanches. In order to further quantify changes in the relative ordering of oscillators during an avalanche, in Fig. 3(b) we compute the "rearrangement fraction," the fraction of oscillators that exchange positions at each time point. We determine this quantity by calculating the normalized Kendall tau distance between cyclic orderings of the oscillators along the unit circle at each pair of successive time points. During a single time step, if all oscillators exchange and permute positions along the unit circle due to rearrangements, the rearrangement fraction will be 1; if no oscillators exchange positions, then this quantity will be 0 .

We observe that, during synchronization, brief peaks in the net force appear and then relax, due to oscillators having sufficient space to rearrange and maximize their spacing. However, as the degree of synchronization increases, oscillators become less likely to have space to freely rearrange, causing repulsive forces to aggregate within the cluster. Eventually, large-scale rearrangement occurs, triggering a cascade of rearrangements that break apart the cluster-visible as a sustained period of nonzero net repulsive force, position exchanges, and dispersion along the unit circle. 


\section{A cellular automaton traffic model recreates the dynamics}

Given our observation that criticality arises due to competing aggregative and dispersive processes occurring over widely separated timescales, we seek to recapitulate our findings in a minimal model that rules out potential alternative mechanisms [57]. The attraction of oscillators towards the mean field qualitatively resembles directed flow in classical traffic models, with steric interactions among oscillators seeding "jams" due to excluded volume effects. We start with the Nagel-Schreckenberg cellular automaton model for traffic on a periodic domain [58]. In this model, $N$ cars are distributed among $M$ sites, represented by a length $M$ string with site values 0 (unoccupied) or 1 (occupied, without overlaps). In each time step, cars at each occupied site move forward a number of units determined by their current velocity and then update their velocity using various acceleration rules (such as random acceleration or braking). A central feature of the model is that a car's velocity is bounded by the number of empty sites preceding it, thus preventing passing - a constraint that triggers the formation of large-scale jams at high densities $N / M \rightarrow 1$, and which allows the system to exhibit self-organized criticality when the density is sufficient for jams to transiently form and then break apart [59]. Here, we modify this model in two ways: (1) We impose an acceleration rule that depends, in part, on the distance of each car from all other cars, and (2) if the net repulsive force on a given car reaches a critical threshold, then the car accelerates at a rate depending on the magnitude and direction of its net force. The latter rule comprises a one-dimensional toppling mechanism for large jams, as occur in classical sandpile models of self-organized criticality [47]. We describe the model in greater detail in Appendix E.

We observe that our modified traffic cellular automaton replicates the irregular buildup and breakup of synchronized states that we observe in the continuous-time oscillator dynamics (Fig. 4). Moreover, the statistical properties of the two models are comparable: The power spectral density of $R$ (calculated by assuming the cars travel on the unit circle) displays $\alpha=1.48 \pm 0.01$, while the raw time series exhibits $H=0.93 \pm 0.01$. The similarity between the oscillator and traffic models underscores the central role of force buildup and rearrangement in determining our observed avalanche dynamics, despite qualitative differences between the two models.

\section{A continuum model maps the critical regime}

That rearrangements trigger cascades indicates the role of interoscillator repulsion in amplifying local events into global cascades. Avalanches therefore are the result of Eq. (1) becoming extremely sensitive to noise (here provided by rearrangements) at intermediate values of $V_{0} / K$. In order to better understand this critical mechanism, we next seek to solve the repulsive oscillator model analytically in the continuum (large $N)$ limit. In this limit, the system comprises an "oscillator fluid" with the dynamical equation

$$
\partial_{t} f+\partial_{\theta}(v f)=0,
$$

where $f(\theta, \omega, t)$ is the probability density of oscillators at position $\theta$ along the unit circle. The force is given by a continuum analog of Eq. (1),

$v=\omega+K \iint f \sin \left(\theta^{\prime}-\theta\right) d \theta^{\prime} d \omega-(1 / N)\left[V_{0}+\xi(t)\right] \partial_{\theta} f$.

The first two terms in this expression follow directly from the Kuramoto model [60]. The remaining term proportional to $\partial_{\theta} f$ follows from the continuum limit of the repulsive interaction (see Appendix B for derivation). Similar density gradient terms appear in continuum versions of critical sandpile models [61]; here, the term helps stabilize the uniform distribution $f=1 /(2 \pi), R=0$. We introduce a heuristic noise force $\xi(t)$ that triggers cascades; we assume that rearrangements are seeded by uncorrelated stochastic events $\left\langle\xi(t) \xi\left(t^{\prime}\right)\right\rangle=$ $\xi_{0} \delta\left(t-t^{\prime}\right)$, with amplitude proportional to the local oscillator density. We assume the simple case of $\xi(t)$ global and uncorrelated, in order to demonstrate that avalanches are not particularly sensitive to details of the noise-however, a more explicit heuristic model of local rearrangements may provide greater similarity to the discrete simulations. Our continuum analysis presented below shows that the simplest way that noise can affect the dynamics of $R(t)$ is through a term of the form $\xi(t) \partial_{\theta} f$, as appears in Eq. (3); other approaches, such as additive or multiplicative noise, do not affect the dynamics of $R(t)$ (see Appendix C).

In order to solve for the dynamics of $R(t)$ subject to Eq. (2), we impose a form for $f(\theta)$ using the Ott-Antonsen ansatz $[60,62]$, which assumes a Fourier representation of the density distribution,

$$
f(\theta)=\frac{g(\omega)}{2 \pi}\left(1+\sum_{n=1}^{\infty} a^{n} e^{i n \theta}+\text { c.c. }\right) .
$$

We insert this equation in Eq. (2), and we assume that $g(\omega)$ is given by a Cauchy distribution of width $\sigma_{\omega}$. The resulting dynamical equation reveals that the full dynamics are captured by those of the order parameter,

$$
\begin{aligned}
\frac{\dot{R}(t)}{R(t)} & =-\sigma_{\omega}-\frac{K}{2}\left[R(t)^{2}-1\right]-\frac{P(R(t))}{2 \pi N}\left[V_{0}+\xi(t)\right], \\
P(R(t)) & \equiv 1+R(t)^{2}+R(t)^{4}+R(t)^{6}+R(t)^{8},
\end{aligned}
$$

where $R(t) e^{i \Phi(t)} \equiv \int e^{i \theta^{\prime}} f\left(\theta^{\prime}\right) d \theta^{\prime}$. In the zero-noise limit ( $\xi=$ $0)$, Eq. (5) admits two physically meaningful solutions,

$$
R=0, \quad R \approx \frac{\sqrt{2}}{4} \sqrt{\sqrt{u^{2}+16(u-1)-16\left(\frac{2 \pi \sigma_{\omega}}{V_{0}}\right)}-u},
$$

where $u \equiv \pi K / V_{0}$. The approximate nonzero solution closely matches the exact solution expressed in terms of the root of the high-degree polynomial $P(R)$ (see Appendix C). Analysis of the eigenvalues associated with these solutions reveals that they exchange stability via a transcritical bifurcation at $V_{0}=$ $\pi N\left(K-2 \sigma_{\omega}\right)$. Discrete oscillator simulations across various $\sigma_{\omega}$ and $V_{0} / K$ show general agreement with Eq. (5) below this critical value (Fig. 5); in this regime the dynamics converge to a partially synchronized state. However, as $V_{0} \rightarrow \pi N(K-$ $\left.2 \sigma_{\omega}\right)$, the dynamics of the discrete simulations undergo an abrupt transition to either stable maximal desynchronization $R=0$ when $\xi_{0}=0$ or avalanche dynamics when $\xi_{0}>0$. 

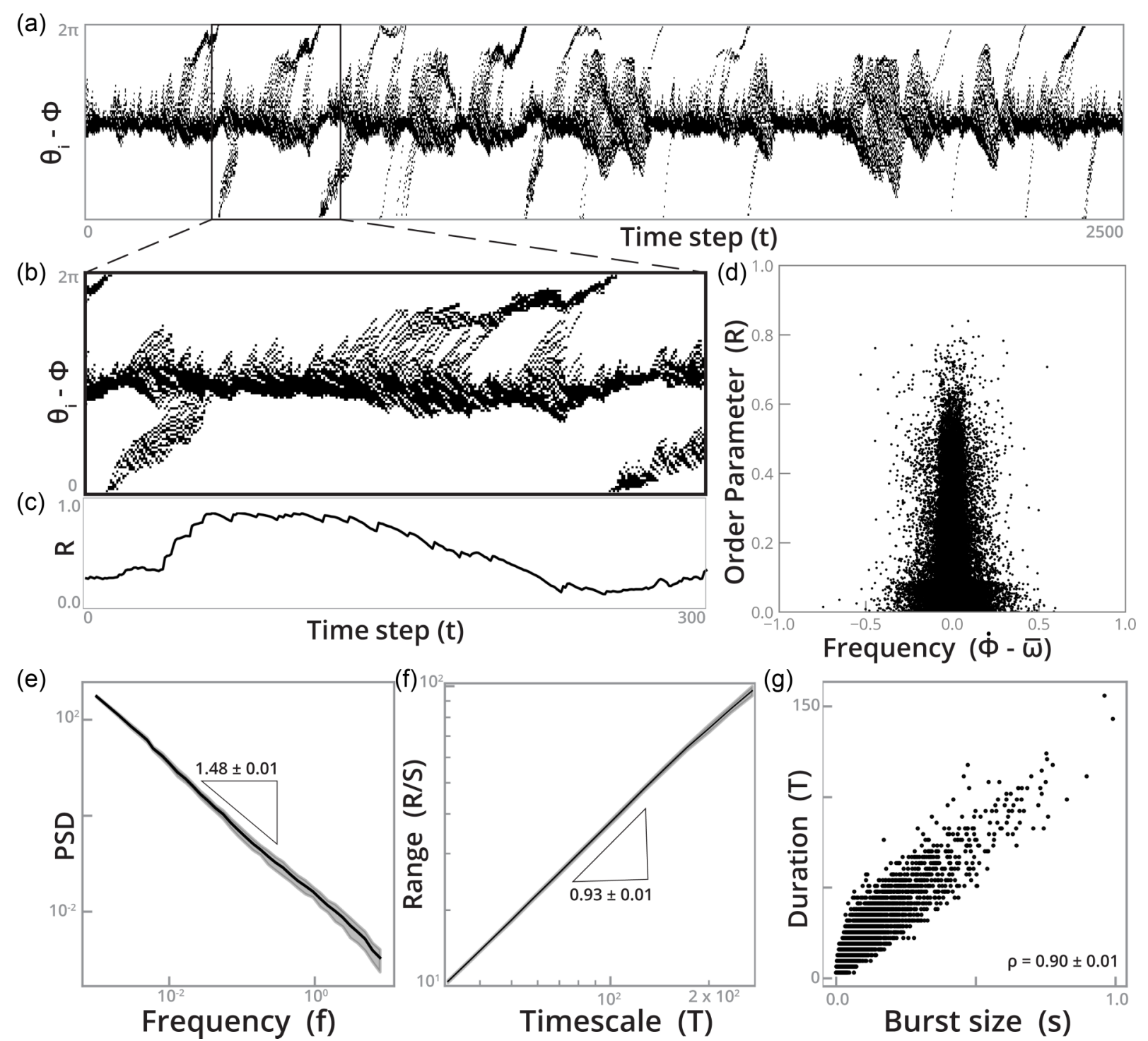

FIG. 4. A cellular automaton traffic model recreates key features of the dynamics. (a) The positions of 60 cars in a toppling cellular automaton traffic model, visualized in a frame rotating with the mean field. (b) A magnified single avalanche event, and (c) the order parameter $R$. (d) $R$ vs the deviation between the mean field and the mean intrinsic frequency. The (e) power spectral density (PSD) and (f) Hurst scaling plot, with power-law exponents annotated, and $(\mathrm{g})$ the topple duration vs size, annotated with the Spearman correlation coefficient. Error ranges comprise bootstrapped standard deviations.

Together, Eqs. (2) and (3) constitute a damped Burgers equation with nonlocal forcing; previous studies have demonstrated that Burgers and Kardar-Parisi-Zhang models can exhibit critical dynamics when driven by noise $[63,64]$. We therefore seek to recreate avalanches observed in our discrete simulations by reintroducing noise $\left(\xi_{0}>0\right)$ into Eq. (3). This produces a stochastic differential equation $d R=F(R) d t+$ $\xi_{0} G(R) d W$, where the deterministic term $F(R)$ is given by the right-hand side of Eq. (5), and $G(R)=-[R(t) / 2 \pi] P(R(t))$ (see Appendix D). We simulate this Stratonovich process for a long duration with different pairs $V_{0} / K$ and $\sigma_{\omega}$, discard a transient period, and then report the long-timescale statistics of $R(t)$ in Fig. 5. We record the mean $R$ to facilitate comparison with Eq. (5), and we record the variance in $R(t)$ to measure the presence and degree of fluctuations in $R$ due to avalanching. We observe that key features of the phase space exactly match between the discrete simulations and theoretical results, despite the relative simplicity of our rearrangement model. Moreover, the analytic stability boundary $V_{0}=$ $\pi N\left(K-2 \sigma_{\omega}\right)$ determines the onset of fluctuations (Fig. 5, dashed red traces), which only occur when the maximally desynchronous state $R=0$ is stable. Thus, while our noise term does not explicitly model small-scale details of rearrangements, it shows that they essentially act as a source of random variation that is amplified by the dynamics at intermediate values of $V_{0} / K$. Differences between the theoretical and observed results are most apparent in the shape and decay of the threshold between partial synchronization and avalanches (or maximal desynchrony when $\xi_{0}=0$ ); a more detailed microscopic rearrangement model would likely resolve these discrepancies. We note that beyond capturing the general form of phase space, the continuum model captures nontrivial properties of our observed avalanches, such as an uptick in the relative amplitude of $R$ fluctuations when $\sigma_{\omega} \approx 0.1-0.3$. 
(a)

(a)

Discrete Simulations
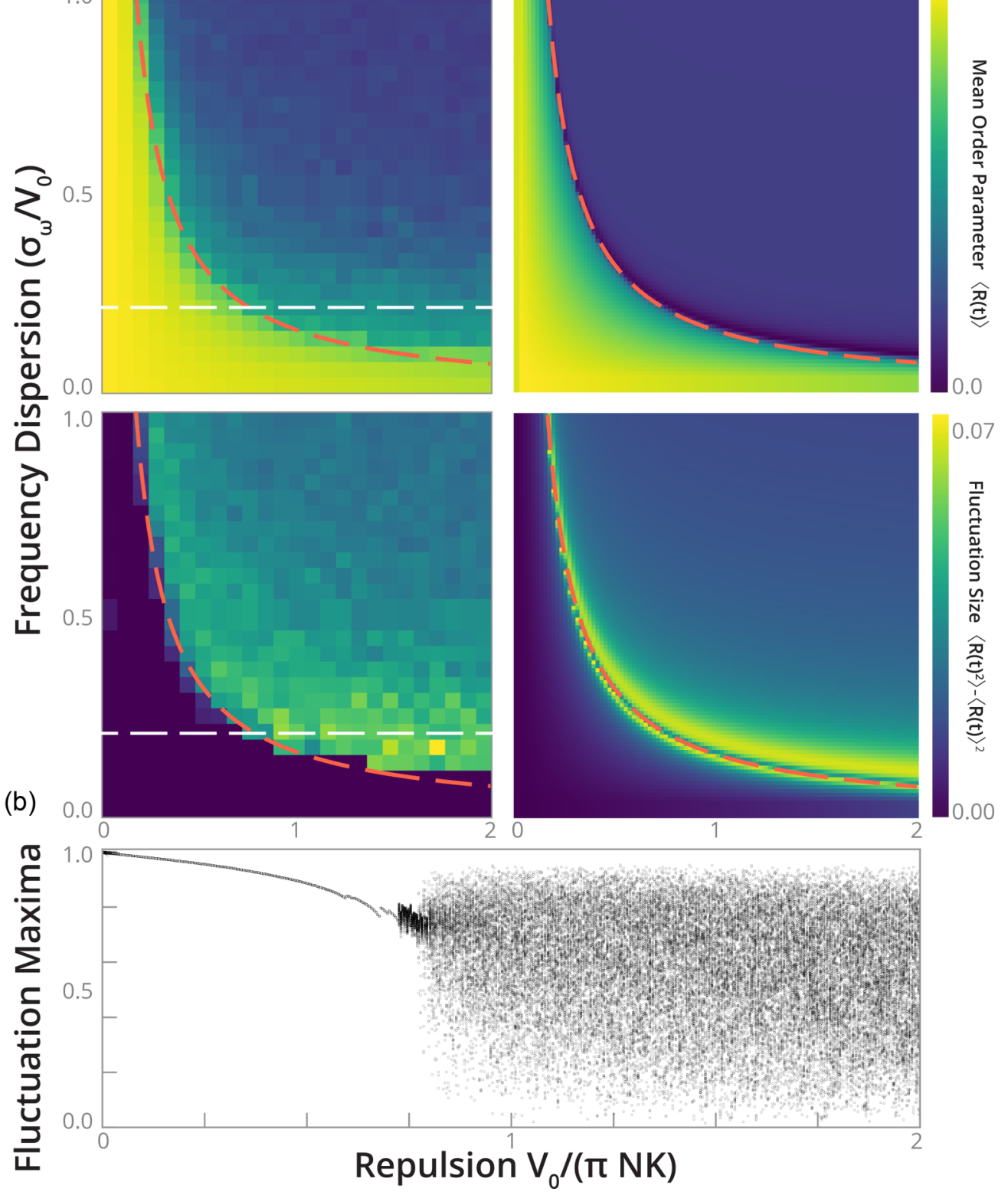

FIG. 5. A stochastic continuum model summarizes the phase space of avalanches. (a) The empirical phase space of fluctuations in $R$ as the standard deviation of oscillator frequencies and relative repulsion strength are varied, and the corresponding phase space for the continuum model with $\xi_{0}=1$. The analytic stability boundary for the continuum model is overlaid (red dashed traces). The white horizontal dashed lines indicate a cross section of the phase space plotted in (b), which shows a bifurcation diagram comprising the extrema taken by $R$ during steady-state fluctuations as $V_{0} /(\pi N K)$ increases.

\section{DISCUSSION}

We have shown that a minimal generalization of classical synchronization models produces unexpectedly rich dynamics, mirroring those of systems exhibiting self-organized criticality and avalanches. We anticipate potential applications of our model to understanding avalanchelike dynamics seen in real-world oscillator networks, ranging from power grids [29], to financial markets [28], to neuronal circuits in the brain [27]. Our observed dynamics exhibit power-law scaling of power spectral density, multifractality, burst buildup, and other characteristic features of self-organized critical phenomena [65]; for example, neuronal avalanches have previously been reported to exhibit power spectral densities with critical exponents between 1 and 2, and Hurst exponent $\sim 0.7$ [53,6669]. However, while the Kuramoto model has frequently been used as a minimal model of neuronal synchronization [8,9], further study is needed in order to determine whether neurons undergoing avalanches can be mapped onto our modified Kuramoto model-particularly because real-world neuronal networks have sophisticated spatial structure and highly nonlinear interactions $[8,35]$. Nonetheless, our results suggest that even neurons with a simple interaction scheme (all-to-all coupling) could produce avalanches when individual neurons have a negative region in their phase-response curve [70], a property that was recently shown to induce complex dynam- 

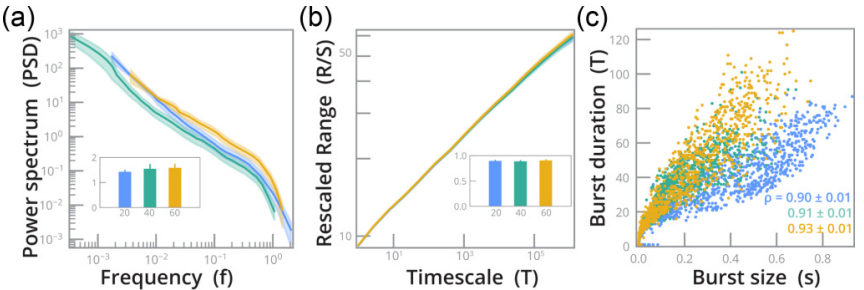

FIG. 6. Dependence of avalanche dynamics on the number of oscillators. (a) The power spectral density, with slopes corresponding to power-law decay exponents shown in the inset, for $R$ from simulations of 20 (blue), 40 (turquoise), and 60 (yellow) oscillators with Gaussian repulsion. (b) A Hurst plot of the rescaled range vs measurement timescale, a measure of fractality for time series, with slopes corresponding to Hurst exponents shown in the inset. (c) Avalanche duration vs size, with Spearman correlations shown in the inset. All error ranges correspond to bootstrapped standard deviations.

ics in experimental oscillator networks [71]. We speculate that, in our model, short-range phase repulsion acts analogously to synaptic depression, in which neurons inhibit one another over short time periods due to local depletion of a neurotransmitter - a mechanism that has previously been shown to be sufficient to produce avalanches with comparable statistical properties to our system [34,35,72]. More broadly, our work provides a minimal example of globally coupled oscillators that produce dynamics poised between synchrony and disorder, illustrating how critical dynamics can increase the sensitivity of real-world oscillator ensembles to noise and external perturbations.

The PYTHON 3 code used to simulate the repulsive oscillator model is available [73].

\section{ACKNOWLEDGMENTS}

We thank Daniel Forger and Iryna Omelchenko for their comments on the manuscript. W.G. was supported by the NSF-Simons Center for Mathematical and Statistical Analysis of Biology at Harvard University, NSF Grant No. DMS 1764269, and the Harvard FAS Quantitative Biology Initiative.

\section{APPENDIX A: NUMERICAL SIMULATIONS AND ANALYSIS TECHNIQUES}

All potential functions are defined on the range $[0,2 \pi]$, with width parameters that scale inversely with $N$. For the triangular potential function, the derivative (comprising two square functions) is calculated and smoothly approximated using a superposition of logistic functions. The qualitative appearance and properties of the avalanches do not vary as the smoothness of the logistic approximation is varied. The results of varying $V_{0}$ and $N$ are shown in Figs. 6 and 7.

Numerical integration is performed using the method of lines. Several solvers and time steps were tested and compared (see Appendix F below); we found best results using a standard LSODA solver as interfaced by SCIPY.INTEGRATE.SOLVE_IVP. Before integrating, we compile

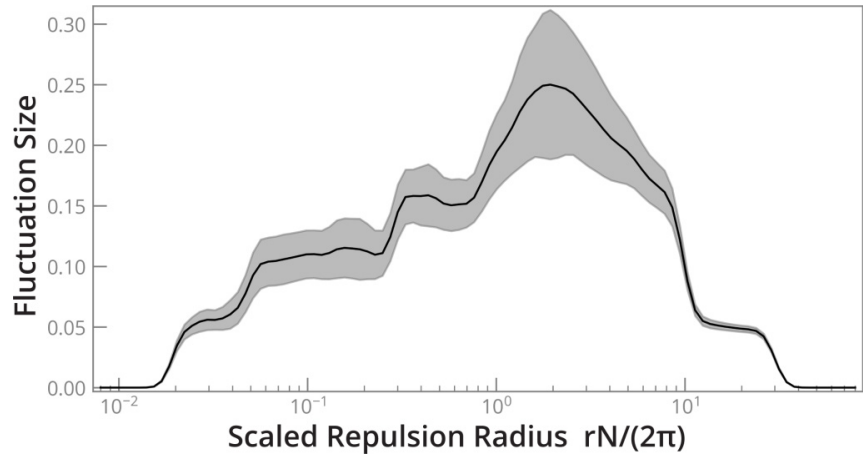

FIG. 7. Dependence of avalanche magnitude on repulsion radius. The amplitude of critical fluctuations as the radius of repulsion is varied.

the right-hand side of the dynamical equation using NUMBA (a just-in-time compiler for PYTHON).

All power-law fits, error ranges, and visualizations are calculated using recommended best-practice techniques based on the cumulative distribution function [74], as implemented in the POWERLAW PYTHON package [75]. We use the default methods recommended for fitting power laws: optimal $x_{\text {min }}$ and $x_{\max }$ are found by computing the minimum KolmogorovSmirnov distance between the data and the fit, and we confirm the quality of our model by performing a goodness-of-fit test between our data and the power-law model, which we compare against stretched exponential and lognormal distributions [74]. All fits were repeated across 500 time series in order to generate error bars.

Hurst exponents and scaling plots are produced using the standard $R / S$ algorithm [76] with random sample consensus (RANSAC) fitting, as implemented in NOLDS [77]. Observed $R / S$ scalings and calculated Hurst exponents were confirmed by comparing against surrogate time series with shuffled data. Equivalent scaling exponents were observed using detrended fluctuation analysis; however, we favor reporting Hurst exponents due to the stationarity of our time series [78] and the relative interpretability of Hurst exponents for multifractal time series.

When calculating avalanche size and duration, we use a numerical peak-finding algorithm to find all extrema in a time series of $R(t)$. We define the size of an event as the difference between a maximum and the next minimum. We define the duration of an avalanche as the number of time points that elapse between two successive minima. We compute Spearman correlations between these two quantities, and we generate error ranges for the reported correlation coefficients by bootstrapping 500 randomly chosen subsets of the time series.

In order to compute the rearrangement fraction as a function of time, we assign each oscillator a unique label and then track the ordering of this set over time. For each pair of adjacent time points, we compute the normalized Kendall tau distance, a measure of the dissimilarity of two ordered sets of equal measure. In order to account for the periodic boundary conditions, we compute this distance not only for the observed orderings, but also for all circular shifts of the orderings, and we use the minimum among all shifts as the normalized cyclic Kendall tau distance. Because our method 
of detecting rearrangements relies on permutations, the resolution matches that of the numerical integration: We will not detect rearrangements on scales shorter than the integration time step. However, because the oscillators are localized to within floating point precision, we expect that all observed rearrangements will be accurate.

\section{APPENDIX B: CONTINUUM LIMIT OF THE SUMMED REPULSIVE INTERACTIONS}

The dynamical equation for $\dot{\theta}_{j}$ contains the term

$$
\frac{1}{N} \sum_{k=1}^{N} \frac{d}{d \theta_{j}} V\left(\theta_{j}, \theta_{k}\right)
$$

where $V\left(\theta_{j}, \theta_{k}\right)=V\left(d\left(\theta_{j}, \theta_{k}\right)\right)$ and $d$ is a distance function on the unit circle that gives the shortest signed angular distance along the unit circle (wrapping around at $\pi$ ), $d\left(\theta_{j}, \theta_{k}\right)=$ $\bmod \left(\theta_{j}-\theta_{k}-\pi, 2 \pi\right)-\pi$.

We take the continuum limit of Eq. (B1), and we assume that $V$ decays sufficiently quickly that $V\left(\theta_{j}, \theta_{k}\right) \rightarrow 0$ as $\mid \theta_{j}-$ $\theta_{k} \mid \rightarrow \pi$, and so $V\left(\theta_{j}, \theta_{k}\right)=V\left(\theta_{j}-\theta_{k}\right)$. Equation (B1) then has the form of a convolution,

$$
\int f\left(\theta^{\prime}\right) \frac{d}{d \theta} V\left(\theta-\theta^{\prime}\right) d \theta^{\prime}
$$

where $f\left(\theta^{\prime}\right)$ is a probability distribution of phases on the interval $[0,2 \pi]$. Next, we note the following property of convolutions:

$$
\frac{d}{d t}(f * g)=f * \frac{d g}{d t}=\frac{d f}{d t} * g .
$$

We therefore exchange the derivatives in Eq. (B2),

$$
\int \frac{d f}{d \theta} V\left(\theta-\theta^{\prime}\right) d \theta^{\prime}
$$

We assume that, in the continuum limit $N \rightarrow \infty, V(\theta-$ $\left.\theta^{\prime}\right) \rightarrow \delta\left(\theta-\theta^{\prime}\right)$, which holds true as long as $V$ is compact and has a width parameter that decreases as $N$ increases [as we require for $V(\cdot)$ in the main text]. For example, if $V$ corresponds to a Gaussian distribution $V=G_{\mu=0, \sigma}$, then this is equivalent to the condition $\sigma=\sigma_{0} / N$ with $\sigma_{0}$ constant. In this case, Eq. (B3) simplifies to $\partial_{\theta} f$. The full continuous-time dynamical equation thus becomes

$$
\begin{aligned}
\partial_{t} f+\partial_{\theta}(v f) & =0, \\
v & \equiv \omega+K \int f\left(\theta^{\prime}\right) \sin \left(\theta^{\prime}-\theta\right) d \theta^{\prime}-\left(V_{0} / N\right) \partial_{\theta} f .
\end{aligned}
$$

The factor of $N$ on the repulsive term appears because we defined the discrete system in the main text extensively: As the number of oscillators $N$ increases, the maximum force attainable by overlapping oscillators also increases by a factor of $N$.

\section{APPENDIX C: OTT-ANTONSEN REDUCTION WITHOUT NOISE}

The dynamics of a continuous distribution of oscillators in the absence of noise is given by

$$
\partial_{t} f+\partial_{\theta}(v f)=0
$$

where

$$
\begin{aligned}
v(\theta, \omega, t)= & \omega+K \int_{-\infty}^{\infty} \int_{0}^{2 \pi} f\left(\theta^{\prime}, \omega, t\right) \sin \left(\theta^{\prime}-\theta\right) d \theta^{\prime} d \omega \\
& -\frac{V_{0}}{N} \partial_{\theta} f(\theta, \omega, t) .
\end{aligned}
$$

We define the order parameter, $z(t)$,

$$
z(t)=\int_{-\infty}^{\infty} \int_{0}^{2 \pi} f\left(\theta^{\prime}, \omega, t\right) e^{i \theta^{\prime}} d \theta^{\prime} d \omega,
$$

for which Eq. (C2) reduces to the form

$$
v(\theta, \omega, t)=\omega+\frac{K}{2 i}\left(z e^{-i \theta}-\bar{z} e^{i \theta}\right)-\frac{V_{0}}{N} \partial_{\theta} f(\theta, \omega, t) .
$$

Following previous applications of the Ott-Antonsen reduction [3,60,79-81], we express the angular distribution of oscillators in terms of its Fourier series expansion

$$
f(\theta, \omega, t)=\frac{g(\omega)}{2 \pi}\left(1+\sum_{n=1}^{\infty} a_{n}(\omega, t) e^{i n \theta}+\bar{a}_{n}(\omega, t) e^{-i n \theta}\right),
$$

where $g(\omega)$ is the distribution of frequencies across the population, $g(\omega)=\int_{0}^{2 \pi} f\left(\theta^{\prime}, \omega, t\right) d \theta^{\prime}$.

The Ott-Antonsen ansatz is a low-order closure for this moment series, $a_{n}(\omega, t)=a(\omega, t)^{n}, \bar{a}_{n}(\omega, t)=\bar{a}(\omega, t)^{n}$. Inserting this ansatz into Eq. (C3), and then inserting the result into Eq. $(\mathrm{C} 1)$, results in the equation

$$
\begin{aligned}
\dot{a}(\omega, t)= & -\frac{a(\omega, t)}{2 \pi}\left(2 \pi i \omega-\pi K+\pi K|a(\omega, t)|^{2}\right. \\
& \left.+\left(V_{0} / N\right) P(|a(t)|)\right),
\end{aligned}
$$

with $P(q) \equiv 1+q^{2}+q^{4}+q^{6}+q^{8}$. Using the definition of the order parameter, we perform the substitution

$$
z(t)=\int_{-\infty}^{\infty} g(\omega) \bar{a}(\omega, t) d \omega, \quad \bar{z}(t)=\int_{-\infty}^{\infty} g(\omega) a(\omega, t) d \omega .
$$

We next choose $g(\omega)$ to be a Cauchy distribution with zero mean $g(\omega)=\left(\sigma_{\omega} / \pi\right)\left[1 /\left(\omega^{2}+\sigma_{\omega}^{2}\right)\right]$. This yields the relation $z(t)=\bar{a}\left(-i \sigma_{\omega}, t\right), \bar{z}(t)=a\left(-i \sigma_{\omega}, t\right)$.

We next apply the substitutions $a\left(-i \sigma_{\omega}, t\right)=$ $R(t) \exp [-i \Phi(t)], \quad \bar{a}\left(-i \sigma_{\omega}, t\right)=R(t) \exp [i \Phi(t)], \quad|a(t)|=$ $R(t)$. The real and imaginary parts of the resulting dynamical equation yield the separate dynamics of $\dot{R}(t), \dot{\Phi}(t)$

$$
\begin{aligned}
& \dot{R}(t)=-\sigma_{\omega} R(t)-\frac{R(t)}{2 \pi}\left(\pi K\left[R(t)^{2}-1\right]+\left(V_{0} / N\right) P(R(t))\right), \\
& \dot{\Phi}(t)=\omega,
\end{aligned}
$$

with high-degree polynomial $P(R(t)) \equiv 1+R(t)^{2}+R(t)^{4}+$ $R(t)^{6}+R(t)^{8}$. Thus, regardless of the value of the order parameter $R(t)$, the oscillators find a state where all corotate at their common frequency $\omega$. 


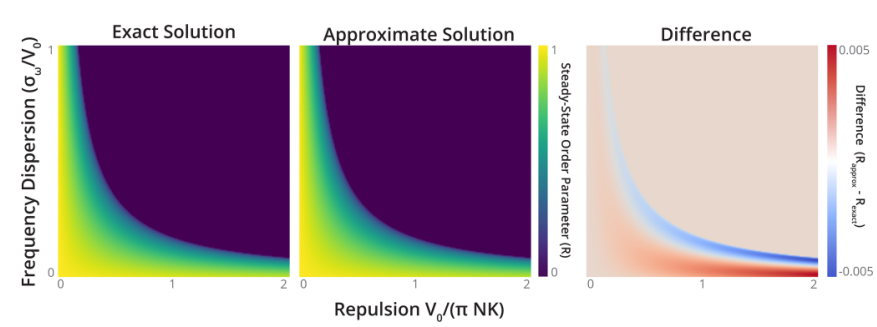

FIG. 8. Exact vs approximate solution to the dynamics without noise. The steady-state solution of the full dynamics, parametrized by $V_{0}$ and $\sigma_{\omega}$, for the exact dynamics and those given by the quartic approximate solution. For these plots, $\xi_{0}=0$.

The steady-state values of the order parameter may be calculated by substituting $\dot{R}(t)=0$ into Eq. (C5)

$$
0=\pi\left(2 \sigma_{\omega}-K\right)+K \pi R(t)^{2}+\left(V_{0} / N\right) P(R(t)) .
$$

The steady states of this equation, as well as the eigenvalues defining their stability, may be solved exactly using existing formulas for the roots of polynomial equations; however, the form of these solutions is not concise, and so we do not reprint them here. These exact solutions predict that $0<R(t)<1$ as $t \rightarrow \infty$ when $V_{0}<\pi N\left(K-2 \sigma_{\omega}\right)$ and that $R(t)=0$ as $t \rightarrow \infty$ when $V_{0}>\pi N\left(K-2 \sigma_{\omega}\right)$. Thus, as the relative strength of the repulsive interaction increases, the steady-state value of the order parameter (and thus degree of synchronization) decreases until reaching zero at $V_{0}=$ $\pi N\left(K-2 \sigma_{\omega}\right)$. As the repulsive interaction further increases, the steady state remains at zero. Thus, at $V_{0}=\pi K N$, the system undergoes a transcritical bifurcation.

To better illustrate the behavior of the full solution, we note that a concise approximate solution may be found by noting that $R(t)^{2}+R(t)^{4}+R(t)^{6}+R(t)^{8} \approx 2 R(t)^{4}$ on the interval $R(t) \in[0,1]$. Performing this substitution into Eq. (C6) results in an approximate solution with the form

$$
R=0, \quad R \approx \frac{\sqrt{2}}{4} \sqrt{\sqrt{u^{2}+16(u-1)-16\left(\frac{2 \pi \sigma_{\omega}}{V_{0}}\right)}-u}
$$

where $u \equiv \pi K / V_{0}$. These solutions have respective eigenvalues

$$
\begin{aligned}
\lambda_{0} & =\frac{1}{2}\left(K-2 \sigma_{\omega}-\frac{V_{0}}{\pi N}\right), \\
\lambda_{1} & =4 \sigma_{\omega}-2 K-\frac{\pi K^{2} N}{8 V_{0}}+\frac{2 V_{0}}{\pi N} \\
& +\frac{N K^{2}}{8 V_{0}} \sqrt{\pi^{2}+\frac{16 \pi V_{0}}{K N}-\frac{16 V_{0}}{K^{2} N}\left(2 \sigma_{\omega} \pi+\frac{V_{0}}{N}\right)} .
\end{aligned}
$$

Both the full and approximate solutions undergo a transcritical bifurcation at $V_{0}=\pi N\left(K-2 \sigma_{\omega}\right)$. Figure 8 shows the full and approximate solutions for several values of $K, V_{0}$, and $\sigma_{\omega}$, illustrating the transcritical bifurcation between partial synchrony and maximal desynchrony.

\section{APPENDIX D: OTT-ANTONSEN REDUCTION WITH NOISE}

\section{Rearrangements as noise proportional to the gradient}

We use a heuristic model of rearrangements as a form of noise acting on the reduced-order dynamics of the oscillators in the continuum limit found above. Because the noise force must affect the dynamics of the order parameter $R(t)$, there are restrictions on how it can enter into the dynamical equations for the evolution of the oscillator phase distribution $f(\theta)$. We emphasize that our noise force $\xi$ does not describe Langevin dynamics of individual oscillators. Rather, we assume deterministic microscale dynamics and then derive a continuum model using the Ott-Antonsen ansatz, and we then introduce the stochastic forcing term $\xi$ acting directly on the mean-field dynamics of $R$, in order to "seed" random toppling events.

We start with the hydrodynamic equation for the time evolution of the oscillator distribution $f(\theta)$, as well as the Ott-Antonsen ansatz for the form of $f(\theta)$,

$$
\partial_{t} f+\partial_{\theta}(v f)=0, \quad f=\frac{1}{2 \pi}\left(1+\sum_{n=1}^{\infty} a^{n} e^{i n \theta}+\text { c.c. }\right) .
$$

We define $v=v_{0}(\theta, t)+v_{\xi}(\theta, t)$, where $v_{0}(\theta, t)$ refers to the forcing on oscillators in the absence of noise,

$$
v_{0}=\omega+\frac{K}{2 i}\left(Z e^{-i \theta}-\bar{Z} e^{i \theta}\right)-\frac{V_{0}}{N} \partial_{\theta} f,
$$

and $v_{\xi}(\theta, t)$ refers to the noise term in the oscillator dynamical equations. In the following sections, we insert this term and various forms of $v_{\xi}(\theta, t)$ into the derivation of Appendix $\mathrm{C}$, in order to determine how various forms of noise affect the dynamics of the order parameter $R(t)$.

Additive case. One option for the presence of noise in Eq. $(\mathrm{C} 1)$ is $v_{\xi}(\theta, t)=\xi(t)$, such that $v=v_{0}+\xi(t)$. In this case, $\dot{R}(t)$ has a form identical to Eq. (C5). The noise solely affects the dynamics of the phase,

$$
\dot{\Phi}(t)=\omega+\xi(t) .
$$

Multiplicative case. Another option for the presence of noise in Eq. (C1) is $v_{\xi}(\theta, t)=f(\theta)^{m} \xi(t)$, such that $v=v_{0}+$ $f(\theta)^{m} \xi(t)$, where $m>0$. For the case of $m=1$, Eq. (C4) has the form

$$
\begin{aligned}
\dot{a}(t)= & -\frac{a(t)}{2 \pi}\left(2 \pi i \omega-\pi K+\pi K|a(t)|^{2}\right. \\
& \left.+\left[V_{0} / N+2 i \xi(t)\right] P(|a(t)|)\right)
\end{aligned}
$$

with high-degree polynomial $P(q) \equiv 1+q^{2}+q^{4}+q^{6}+q^{8}$. This corresponds to $\dot{R}(t)$ having an identical form to Eq. (C5), while the phase $\Phi(t)$ has dynamics given by

$$
\dot{\Phi}(t)=\omega+\frac{\xi(t)}{\pi}\left[1+R(t)^{2}+R(t)^{4}+R(t)^{6}+R(t)^{8}\right] .
$$

A similar derivation may be used to show that the dynamics of $R(t)$ are unaffected by multiplicative noise to arbitrary order $m$.

Gradient case. A final option for the presence of noise in Eq. (C1) is $v_{\xi}(\theta, t)=\frac{\partial^{m} f(\theta)}{\partial \theta^{m}} \xi(t)$, such that $v=v_{0}+$ $\frac{\partial^{m} f(\theta)}{\partial \theta^{m}} \xi(t)$, where $m>0$. For the case of $m=1$, Eq. (C4) has 
the form

$$
\begin{aligned}
\dot{a}(t)= & -\frac{a(t)}{2 \pi}\left(2 \pi i \omega-\pi K+\pi K|a(t)|^{2}\right. \\
& \left.+\left[V_{0} / N+\xi(t)\right] P(|a(t)|)\right)
\end{aligned}
$$

with high-degree polynomial $P(q) \equiv 1+q^{2}+q^{4}+q^{6}+q^{8}$. This corresponds to dynamics given by

$$
\begin{aligned}
\dot{R}(t)= & -\sigma_{\omega} R(t)-\frac{R(t)}{2 \pi}\left(\pi K\left[R(t)^{2}-1\right]\right. \\
& \left.+\left[V_{0} / N+\xi(t)\right] P(R(t))\right), \\
\dot{\Phi}(t)= & \omega .
\end{aligned}
$$

Thus we find that the simplest manner in which noise can affect the dynamics of $R(t)$ is through a term proportional to the phase density gradient.

\section{Derivation of stochastic differential equation}

Based on our analysis of the dynamical equations above, we assume that the noise enters the dynamical equations through a term of the form $\frac{\partial f(\theta)}{\partial \theta} \xi(t)$. As seen above, this is equivalent to making the substitution $V_{0} / N \rightarrow\left[V_{0} / N+\xi(t)\right]$ in the dynamical equations. Isolating terms in $\dot{R}(t)$ by their order in $\xi(t)$ reveals that the dynamics have the form of a Stratonovich process,

$$
d R=F(R) d t+\xi_{0} G(R) d W,
$$

where $\xi_{0}$ is a noise amplitude term and

$$
\begin{aligned}
& F(R)=-\sigma_{\omega} R(t)-\frac{R(t)}{2 \pi}\left(\pi K\left[R(t)^{2}-1\right]+\left(V_{0} / N\right) P(R(t))\right), \\
& G(R)=-\frac{R(t)}{2 \pi} P(R(t)) .
\end{aligned}
$$

with high-degree polynomial $P(R(t)) \equiv 1+R(t)^{2}+R(t)^{4}+$ $R(t)^{6}+R(t)^{8}$. Note that the first "drift" term is identical to the dynamical equation for the zero-noise limit, Eq. (C5). For this study, we assume a standard Langevin noise term, $\left\langle\xi(t) \xi\left(t^{\prime}\right)\right\rangle=\xi_{0} \delta\left(t-t^{\prime}\right)$, making $W$ a Wiener process. Using these definitions, we numerically simulate Eq. (D2) using the Euler-Maruyama method.

\section{APPENDIX E: CELLULAR AUTOMATON TRAFFIC MODEL}

Our cellular automaton model is a modified version of the Nagel-Schreckenberg traffic model $[58,59,82]$. The original model evolves on a one-dimensional ring of $L$ sites, each of which can be either occupied (1) or unoccupied (0). Each occupied site represents a "car," and cars cannot overlap or overtake one another. In the original model, fluctuations in the speed of one car (due to random braking events, for example) provoke the spontaneous formation of large jams of cars positioned bumper to bumper within the circular domain. The jam breaks up only when enough time has elapsed for cars at the head of the jam to accelerate away from the jam.

To capture our hypothesized mechanism for jamming in the repulsive oscillator model, we modify the NagelSchreckenberg model in two key ways: (1) Cars travel in motorcades in which all cars are attracted to the mean location of all cars, even if it is behind them. (2) In high-density regions, "toppling" events occur that are the one-dimensional equivalent of topples in classical sandpile models of criticality [47]. For each car in the motorcade, if the number of cars directly afore it exceed those directly behind it (or vice versa), then the car has some probability of moving to the nearest unoccupied lattice site. This probability increases as the difference in afore and aft cars increases. Overall, this probabilistic toppling rule causes cars located in regions of a jam with a high pressure differential to locally rearrange their positions within the jam more frequently, by analogy to gradient-driven rearrangements among oscillators in our repulsive oscillator simulations.

All together, our cellular automaton model comprises the following steps:

(a) Initialization. For a given density parameter $\rho, M=$ floor $(\rho L)$ sites are randomly chosen to have cars at the start of the simulation. A set of random, integer velocities $\left\{v_{i}\right\}, v_{i} \in$ $\left\{0,1, \ldots, v_{\max }\right\}$, is assigned to the set of cars. In subsequent time steps, the locations of all of the cars are then updated according to the following rules.

(b) Iteration. Within each time step of the model, the following steps are performed:

(b1) Calculation of the mean location. The mean location of all $M$ cars, $\bar{x}$, is calculated. For each car $i$, the number of sites $d_{i}$ between its location $x_{i}$ and the mean location $\bar{x}$ is then calculated. Periodic boundary conditions are assumed; if the forward distance along the circle is more than $L / 2$ sites, then the negative backward distance is used. Therefore $d_{i} \in[-L / 2, L / 2]$.

(b2) Mean-field velocity update. The velocity of each car, $v_{i}$, is updated using the following relation: $v_{i} \leftarrow(1-s) v_{i}+$ $s d_{i}$. The synchronization parameter $s$ is treated as a model parameter: If $s=0$, then the mean location does not affect the cars' velocities at all; however, if $s=1$, then the cars always instantaneously update their velocity to their distance from the mean location.

(b3) Toppling of large jams. From the list of $x$ positions, jams are identified as sequences of consecutive 1 values in the ring of $L$ car positions. These jams are "toppled" according to the following rules:

(i) In each jam, the pressure gradient on each car in the jam, $\partial p_{i}$, is calculated as the number of cars behind it in the jam minus the number of cars in front of it in the jam. For example, the jam corresponding to the sequence $0,1,1,1,1,1,0$ corresponds to a sequence of pressure gradient values $0,-4,-3,0,3,4,0$. In order to remain physical, a cutoff parameter is imposed, which corresponds to a maximum radius over which to impose the gradient. For example, with a cutoff radius of 2 , the pressure gradient of the jam $0,1,1,1,1,1,0$ is $0,-2,-1,0,1,2,0$.

(ii) A random subset is chosen of all the cars that currently exceed a threshold $\left|\partial p_{i}\right|>p_{\text {thresh }}$. Cars with larger pressure gradients have a larger probability of being chosen. We note that the value of $p_{\text {thresh }}$ sets the average frequency and amplitude of toppling events but does not otherwise affect the dynamics.

(iii) Among all cars in the random subset, the velocity of each car in the jam is increased by an amount proportional to 


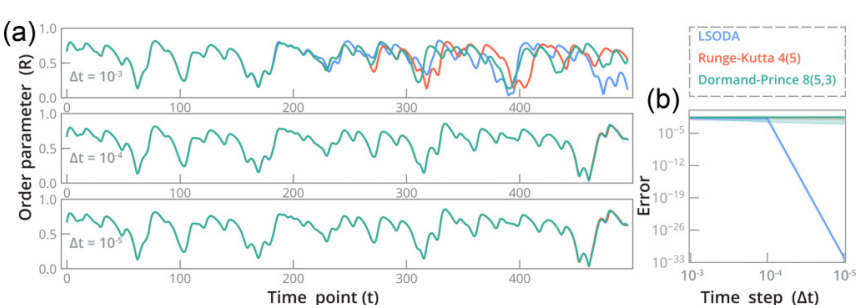

FIG. 9. Numerical robustness of avalanche results. (a) Example trajectories of the Kuramoto order parameter, for different integration methods (colors) and for different maximum time steps (panels). Trajectories for the LSODA and Runge-Kutta methods are obscured by the Dormand-Prince trajectory in the lower two panels, indicating strong agreement. (b) Mean-square error between the observed trajectories, and a fiducial trajectory calculated with maximum time step of $\Delta t=10^{-7}$. Error bars correspond to replicates with different initial conditions. For this panel, $N=40, K=0.4, V_{0}=0.8$, $\sigma_{\omega}=0.2$.

the pressure gradient, $v_{i} \leftarrow v_{i}+f \partial p_{i}$, where $f$ is the amplitude of the rearrangement force.

(b4) Acceleration and maximum velocity. The speeds of all cars are increased by $1, v_{i} \leftarrow v_{i}+1$. Any cars that have a velocity greater than the maximum velocity are reset to the maximum velocity, $v_{i} \leftarrow \min \left\{v_{i}, v_{\max }\right\}$.

(b5) Braking to avoid collisions. The distance between each car and the next car in front of it is calculated in order to produce a set of spacings $\eta_{i}$. For cars that have a velocity larger than the spacing, the velocity is reset to equal the spacing, $v_{i} \leftarrow \min \left\{\eta_{i}, v_{\max }\right\}$. Following the original NagelSchreckenberg model, among the cars that brake, a random subset is chosen to "overbrake" by 1 unit, $v_{i}^{\text {samp }} \leftarrow v_{i}^{\text {samp }}-1$, with probability $p_{\text {brake }}$.

(b6) Position update. The positions of all cars are updated using the final value of the velocity, $x_{i} \leftarrow x_{i}+v_{i}$.

The primary parameters that govern the behavior of the model are the density $\rho$, the synchronization parameter $s$, the magnitude of the critical force $p_{\text {thresh }}$, and the fraction of cars that overbrake, $p_{\text {brake }}$. Consistent with the original Nagel-Schreckenberg model, if $p_{\text {thresh }}$ approaches 1 , the size and duration of jams increase.

\section{APPENDIX F: NUMERICAL STABILITY OF AVALANCHES}

Due to the separation of timescales between synchronization and repulsion in our system, we consider the degree to which our observed dynamics are robust to details of the numerical integration scheme, in order to rule out numerical artifacts. We compute an expensive fiducial trajectory using an integration time step of $\Delta t=10^{-7}$, and we use it to assess the accuracy of trajectories generated with different numerical integrators and time steps (Fig. 9). Across three different integrators (two fixed-step, one variable-step), we find that long-timescale simulations are consistent as long as the maximum time step is less than $10^{-4}$, and that the accuracy is highest for LSODA.
[1] J. C. González-Avella, M. G. Cosenza, and M. San Miguel, Localized coherence in two interacting populations of social agents, Physica A (Amsterdam) 399, 24 (2014).

[2] F. Molnar, T. Nishikawa, and A. E. Motter, Asymmetry underlies stability in power grids, Nat. Commun. 12, 1457 (2021).

[3] M. J. Panaggio, and D. M. Abrams, Chimera states: Coexistence of coherence and incoherence in networks of coupled oscillators, Nonlinearity 28, R67 (2015).

[4] A. Pikovsky, M. Rosenblum, and J. Kurths, Synchronization: A Universal Concept in Nonlinear Sciences, Cambridge Nonlinear Science Series Vol. 12 (Cambridge University Press, New York, 2003).

[5] S. Li, R. Batra, D. Brown, H.-D. Chang, N. Ranganathan, C. Hoberman, D. Rus, and H. Lipson, Particle robotics based on statistical mechanics of loosely coupled components, Nature (London) 567, 361 (2019).

[6] A. T. Winfree, Biological rhythms and the behavior of populations of coupled oscillators, J. Theor. Biol. 16, 15 (1967).

[7] Y. Kuramoto, Chemical Oscillations, Waves, and Turbulence (Springer, New York, 1984).

[8] C. W. Lynn and D. S. Bassett, The physics of brain network structure, function and control, Nat. Rev. Phys. 1, 318 (2019).

[9] J. Cabral, E. Hugues, O. Sporns, and G. Deco, Role of local network oscillations in resting-state functional connectivity, Neuroimage 57, 130 (2011).

[10] T. Danino, O. Mondragón-Palomino, L. Tsimring, and J. Hasty, A synchronized quorum of genetic clocks, Nature (London) 463, 326 (2010).
[11] A. Vilfan and F. Jülicher, Hydrodynamic Flow Patterns and Synchronization of Beating Cilia, Phys. Rev. Lett. 96, 058102 (2006).

[12] W. Gilpin, M. S. Bull, and M. Prakash, The multiscale physics of cilia and flagella, Nat. Rev. Phys. 2, 74 (2020).

[13] Y. Zhang and A. E. Motter, Mechanism for Strong Chimeras, Phys. Rev. Lett. 126, 094101 (2021).

[14] C. Kuehn and C. Bick, A universal route to explosive phenomena, Sci. Adv. 7, eabe3824 (2021).

[15] M. Fruchart, R. Hanai, P. B. Littlewood, and V. Vitelli, Non-reciprocal phase transitions, Nature (London) 592, 363 (2021).

[16] N. Y. Yao, C. Nayak, L. Balents, and M. P. Zaletel, Classical discrete time crystals, Nat. Phys. 16, 438 (2020).

[17] Y. Kuramoto and D. Battogtokh, Coexistence of coherence and incoherence in nonlocally coupled phase oscillators, Nonlinear Phenom. Complex Syst. 5, 380 (2002).

[18] D. M. Abrams and S. H. Strogatz, Chimera States for Coupled Oscillators, Phys. Rev. Lett. 93, 174102 (2004).

[19] B. Ottino-Löffler and S. H. Strogatz, Volcano Transition in a Solvable Model of Frustrated Oscillators, Phys. Rev. Lett. 120, 264102 (2018).

[20] A. Zakharova, M. Kapeller, and E. Schöll, Chimera Death: Symmetry Breaking in Dynamical Networks, Phys. Rev. Lett. 112, 154101 (2014).

[21] P. A. Tass, Phase Resetting in Medicine and Biology: Stochastic Modelling and Data Analysis (Springer, New York, 2007). 
[22] O. V. Popovych, C. Hauptmann, and P. A. Tass, Effective Desynchronization by Nonlinear Delayed Feedback, Phys. Rev. Lett. 94, 164102 (2005).

[23] H. Hong and S. H. Strogatz, Conformists and contrarians in a Kuramoto model with identical natural frequencies, Phys. Rev. E 84, 046202 (2011).

[24] L. Tsimring, N. Rulkov, M. Larsen, and M. Gabbay, Repulsive Synchronization in an Array of Phase Oscillators, Phys. Rev. Lett. 95, 014101 (2005).

[25] V. Nicosia, M. Valencia, M. Chavez, A. Díaz-Guilera, and V. Latora, Remote Synchronization Reveals Network Symmetries and Functional Modules, Phys. Rev. Lett. 110, 174102 (2013).

[26] E. Omel'chenko, Y. L. Maistrenko, and P. A. Tass, Chimera States: The Natural Link between Coherence and Incoherence, Phys. Rev. Lett. 100, 044105 (2008).

[27] J. M. Beggs and D. Plenz, Neuronal avalanches in neocortical circuits, J. Neurosci. 23, 11167 (2003).

[28] L. Bellenzier, J. V. Andersen, and G. Rotundo, Contagion in the world's stock exchanges seen as a set of coupled oscillators, Econ. Model. 59, 224 (2016).

[29] A. E. Motter, S. A. Myers, M. Anghel, and T. Nishikawa, Spontaneous synchrony in power-grid networks, Nat. Phys. 9, 191 (2013).

[30] J. A. Acebrón, L. L. Bonilla, C. J. P. Vicente, F. Ritort, and R. Spigler, The Kuramoto model: A simple paradigm for synchronization phenomena, Rev. Mod. Phys. 77, 137 (2005).

[31] W. Zhou, Z. Hao, and N. Gravish, Collective synchronization of undulatory movement through contact, arXiv:2012.07124.

[32] D. Levis, I. Pagonabarraga, and A. Díaz-Guilera, Synchronization in Dynamical Networks of Locally Coupled Self-Propelled Oscillators, Phys. Rev. X 7, 011028 (2017).

[33] G. Gogia and J. C. Burton, Emergent Bistability and Switching in a Nonequilibrium Crystal, Phys. Rev. Lett. 119, 178004 (2017).

[34] A. Levina, J. M. Herrmann, and T. Geisel, Dynamical synapses causing self-organized criticality in neural networks, Nat. Phys. 3, 857 (2007).

[35] D. R. Chialvo, Emergent complex neural dynamics, Nat. Phys. 6, 744 (2010).

[36] H. Hong and S. H. Strogatz, Kuramoto Model of Coupled Oscillators with Positive and Negative Coupling Parameters: An Example of Conformist and Contrarian Oscillators, Phys. Rev. Lett. 106, 054102 (2011).

[37] S. Heitmann and G. B. Ermentrout, Synchrony, waves and ripple in spatially coupled Kuramoto oscillators with Mexican hat connectivity, Biol. Cybern. 109, 333 (2015).

[38] P. S. Skardal and A. Arenas, Abrupt Desynchronization and Extensive Multistability in Globally Coupled Oscillator Simplexes, Phys. Rev. Lett. 122, 248301 (2019).

[39] P. S. Skardal and A. Arenas, Higher order interactions in complex networks of phase oscillators promote abrupt synchronization switching, Commun. Phys. 3, 218 (2020).

[40] H. Daido, Critical conditions of macroscopic mutual entrainment in uniformly coupled limit-cycle oscillators, Prog. Theor. Phys. 89, 929 (1993).

[41] C. Bick, M. Timme, D. Paulikat, D. Rathlev, and P. Ashwin, Chaos in Symmetric Phase Oscillator Networks, Phys. Rev. Lett. 107, 244101 (2011).

[42] C. Bick, Heteroclinic switching between chimeras, Phys. Rev. E 97, 050201(R) (2018).
[43] P. Clusella and A. Politi, Irregular collective dynamics in a Kuramoto-Daido system, J. Phys.: Complex. 2, 014002 (2020).

[44] M. Rosenblum and A. Pikovsky, Self-Organized Quasiperiodicity in Oscillator Ensembles with Global Nonlinear Coupling, Phys. Rev. Lett. 98, 064101 (2007).

[45] E. A. Martens, S. Thutupalli, A. Fourrière, and O. Hallatschek, Chimera states in mechanical oscillator networks, Proc. Natl. Acad. Sci. USA 110, 10563 (2013).

[46] Z. G. Nicolaou, D. Eroglu, and A. E. Motter, Multifaceted Dynamics of Janus Oscillator Networks, Phys. Rev. X 9, 011017 (2019).

[47] P. Bak, C. Tang, and K. Wiesenfeld, Self-Organized Criticality: An Explanation of the $1 / f$ Noise, Phys. Rev. Lett. 59, 381 (1987).

[48] H. J. Jensen, K. Christensen, and H. C. Fogedby, $1 / f$ noise, distribution of lifetimes, and a pile of sand, Phys. Rev. B 40, 7425 (1989).

[49] G. A. Held, D. H. Solina, H. Solina, D. T. Keane, W. J. Haag, P. M. Horn, and G. Grinstein, Experimental Study of CriticalMass Fluctuations in an Evolving Sandpile, Phys. Rev. Lett. 65 1120 (1990).

[50] H. J. S. Feder and J. Feder, Self-Organized Criticality in a StickSlip Process, Phys. Rev. Lett. 66, 2669 (1991).

[51] H. Kantz and T. Schreiber, Nonlinear Time Series Analysis, Cambridge Nonlinear Science Series Vol. 7 (Cambridge University Press, New York, 2004).

[52] P. Kapiris, K. Eftaxias, and T. Chelidze, Electromagnetic Signature of Prefracture Criticality in Heterogeneous Media, Phys. Rev. Lett. 92, 065702 (2004).

[53] N. Friedman, S. Ito, B. A. W. Brinkman, M. Shimono, R. E. Lee DeVille, K. A. Dahmen, J. M. Beggs, and T. C. Butler, Universal Critical Dynamics in High Resolution Neuronal Avalanche Data, Phys. Rev. Lett. 108, 208102 (2012).

[54] M. Wolfrum and O. E. Omel'chenko, Chimera states are chaotic transients, Phys. Rev. E 84, 015201(R) (2011).

[55] L.-W. Kong and Y.-C. Lai, Scaling law of transient lifetime of chimera states under dimension-augmenting perturbations, Phys. Rev. Research 2, 023196 (2020).

[56] J. Van Hemmen and W. Wreszinski, Lyapunov function for the Kuramoto model of nonlinearly coupled oscillators, J. Stat. Phys. 72, 145 (1993).

[57] G. Boffetta, V. Carbone, P. Giuliani, P. Veltri, and A. Vulpiani, Power Laws in Solar Flares: Self-Organized Criticality or Turbulence? Phys. Rev. Lett. 83, 4662 (1999).

[58] K. Nagel and M. Schreckenberg, A cellular automaton model for freeway traffic, J. Phys. I 2, 2221 (1992).

[59] K. Nagel and M. Paczuski, Emergent traffic jams, Phys. Rev. E 51, 2909 (1995).

[60] E. Ott and T. M. Antonsen, Low dimensional behavior of large systems of globally coupled oscillators, Chaos 18, 037113 (2008).

[61] L. Gil and D. Sornette, Landau-Ginzburg Theory of SelfOrganized Criticality, Phys. Rev. Lett. 76, 3991 (1996).

[62] C. Bick, M. Goodfellow, C. R. Laing, and E. A. Martens, Understanding the dynamics of biological and neural oscillator networks through exact mean-field reductions: A review, J. Math. Neurosci. 10, 2190 (2020).

[63] P. H. Diamond and T. Hahm, On the dynamics of turbulent transport near marginal stability, Phys. Plasmas 2, 3640 (1995). 
[64] G. Szabó, M. Alava, and J. Kertész, Self-organized criticality in the Kardar-Parisi-Zhang equation, Europhys. Lett. 57, 665 (2002).

[65] J. Mallinson, S. Shirai, S. K. Acharya, S. K. Bose, E. Galli, and S. A. Brown, Avalanches and criticality in self-organized nanoscale networks, Sci. Adv. 5, eaaw8438 (2019).

[66] J. M. Palva, A. Zhigalov, J. Hirvonen, O. Korhonen, K. Linkenkaer-Hansen, and S. Palva, Neuronal long-range temporal correlations and avalanche dynamics are correlated with behavioral scaling laws, Proc. Natl. Acad. Sci. USA 110, 3585 (2013).

[67] A. J. Fontenele, N. A. P. de Vasconcelos, T. Feliciano, L. A. A. Aguiar, C. Soares-Cunha, B. Coimbra, L. Dalla Porta, S. Ribeiro, A. J. Rodrigues, N. Sousa, P. V. Carelli, and M. Copelli, Criticality between Cortical States, Phys. Rev. Lett. 122, 208101 (2019).

[68] J. Pu, H. Gong, X. Li, and Q. Luo, Developing neuronal networks: Self-organized criticality predicts the future, Sci. Rep. 3, 1081 (2013).

[69] M. Jannesari, A. Saeedi, M. Zare, S. Ortiz-Mantilla, D. Plenz, and A. A. Benasich, Stability of neuronal avalanches and longrange temporal correlations during the first year of life in human infants, Brain Struct. Funct. 225, 1169 (2020).

[70] R. Dodla and C. J. Wilson, Effect of phase response curve shape and synaptic driving force on synchronization of coupled neuronal oscillators, Neural Comput. 29, 1769 (2017).

[71] D. Călugăru, J. F. Totz, E. A. Martens, and H. Engel, First-order synchronization transition in a large population of strongly coupled relaxation oscillators, Sci. Adv. 6, eabb2637 (2020).
[72] D. Pazó and E. Montbrió, From Quasiperiodic Partial Synchronization to Collective Chaos in Populations of Inhibitory Neurons with Delay, Phys. Rev. Lett. 116, 238101 (2016).

[73] https://github.com/williamgilpin/kurep_paper.

[74] A. Clauset, C. R. Shalizi, and M. E. Newman, Power-law distributions in empirical data, SIAM Rev. 51, 661 (2009).

[75] J. Alstott, E. Bullmore, and D. Plenz, powerlaw: a python package for analysis of heavy-tailed distributions, PloS One $\mathbf{9}$, e85777 (2014).

[76] B. B. Mandelbrot and J. R. Wallis, Robustness of the rescaled range $R / S$ in the measurement of noncyclic long run statistical dependence, Water Resour. Res. 5, 967 (1969).

[77] C. Schölzel, Nonlinear measures for dynamical systems, 2019, doi: 10.5281/zenodo.3814723.

[78] C.-K. Peng, S. V. Buldyrev, S. Havlin, M. Simons, H. E. Stanley, and A. L. Goldberger, Mosaic organization of DNA nucleotides, Phys. Rev. E 49, 1685 (1994).

[79] D. M. Abrams, R. Mirollo, S. H. Strogatz, and D. A. Wiley, Solvable Model for Chimera States of Coupled Oscillators, Phys. Rev. Lett. 101, 084103 (2008).

[80] E. A. Martens, E. Barreto, S. H. Strogatz, E. Ott, P. So, and T. M. Antonsen, Exact results for the Kuramoto model with a bimodal frequency distribution, Phys. Rev. E 79, 026204 (2009).

[81] T. Kotwal, X. Jiang, and D. M. Abrams, Connecting the Kuramoto Model and the Chimera State, Phys. Rev. Lett. 119, 264101 (2017).

[82] M. Paczuski and K. Nagel, Self-organized criticality and $1 / f$ noise in traffic, in Traffic and Granular Flow, edited by D. E. Wolf, M. Schreckenberg, and A. Bachem (World Scientific, Singapore, 1996), p. 73-85. 